\title{
Peritonealkarzinose: Präoperative Diagnostik und Patientenselektion
}

\author{
Ingmar Königsrainer ${ }^{\mathrm{a}}$ Florian Struller ${ }^{\mathrm{a}}$ Philipp Horvath $^{\mathrm{a}}$ Bernhard Klumpp ${ }^{\mathrm{b}}$ \\ Alfred Königsrainer ${ }^{\mathrm{a}}$ Stefan Beckert ${ }^{\mathrm{a}}$ \\ ${ }^{a}$ Klinik für Allgemeine, Viszeral- und Transplantationschirurgie, \\ ${ }^{\mathrm{b}}$ Klinik für Diagnostische und Interventionelle Radiologie, Comprehensive Cancer Center, Universität Tübingen, Deutschland
}

\section{Schlüsselwörter}

Peritonealkarzinose $\cdot$ Hypertherme intraperitoneale Chemotherapie, HIPEC - Selektion - Diagnostik

\section{Zusammenfassung}

Hintergrund: Die zytoreduktive Chirurgie und die hypertherme intraperitoneale Chemotherapie spielen inzwischen eine zunehmend wichtige Rolle in der Therapie der Peritonealkarzinose bei zahlreichen Tumorentitäten. Methode: Die wichtigsten Selektionskriterien werden erläutert. Ergebnisse: Neben dem Alter und Allgemeinzustand sowie der Auswahl geeigneter Tumorentitäten spielt die Tumorlast, die möglichst exakt bereits vor der Operation abgeschätzt werden sollte, eine entscheidende Rolle. Schlussfolgerung: Entscheidend ist eine Selektion geeigneter Patienten, um den onkologischen Benefit zu steigern und die Morbidität zu verringern.

\section{Einleitung}

Die Peritonealkarzinose tritt als besondere Form der Metastasierung im Rahmen verschiedener gastrointestinaler und gynäkologischer Tumorerkrankungen auf. Die Prognose der betroffenen Patienten ist insgesamt ungünstig [1,2]. Ein vielversprechendes Therapiekonzept mit einem potenziell kurativen Ansatz ist die zytoreduktive Chirurgie, die häufig multiviszerale Resektionen beinhaltet, kombiniert mit einer hyperthermen intraperitonealen Chemotherapie (HIPEC) [3].

Um das Ziel eines möglichst langen tumorfreien Überlebens zu erreichen, ist eine vollständige (CC-0) oder annä-

\section{Keywords}

Peritoneal carcinomatosis - Hyperthermic intraperitoneal chemotherapy, HIPEC · Selection · Diagnostics

\section{Summary}

Peritoneal Carcinomatosis: Preoperative Diagnostics and Patient Selection

Background: Cytoreductive surgery and hyperthermic intraperitoneal chemotherapy is nowadays considered as an important multimodal treatment approach in patients suffering from peritoneal surface malignancy. Method: The relevant selection criteria are discussed in this article. Results: Selection criteria include age and general performance status as well as the estimation of the preoperative tumor load, which is relevant for long-term prognosis. Conclusion: Patient selection, however, still remains the Achilles heel regarding the improvement of oncologic outcome and the reduction of morbidity.

hernd vollständige (CC-1) Zytoreduktion erforderlich [4]. Nicht sichtbare Tumorknoten oder solche kleiner als 2,5 mm werden dann potenziell von der HIPEC erfasst.

Entscheidend ist jedoch eine optimale Patientenselektion, um jene Patienten auszuwählen, die potenziell von diesem multimodalen Eingriff profitieren können, und um anderen Patienten einen «unnötigen» Eingriff möglichst zu ersparen. Dabei haben neben der Auswahl der laut bisherigen Erfahrungen geeigneten Tumorentitäten wie dem Mesotheliom, Pseudomyxoma peritonei und kolorektalen Karzinom insbesondere der Allgemeinzustand des Patienten, die individuelle Tumorbiologie und die präoperative Tumorlast die wichtigste Bedeutung.

\begin{tabular}{ll}
\hline KARGER & ( ) 2013 S. Karger GmbH, Freiburg \\
1662-6664/13/0294-0208\$38.00/0 \\
$\begin{array}{l}\text { Fax +49 761 452 07 14 } \\
\text { Information@Karger.com } \\
\text { www.karger.com }\end{array}$ & $\begin{array}{l}\text { Accessible online at: } \\
\text { www.karger.com/vim }\end{array}$
\end{tabular}




\section{Allgemeinzustand und Alter}

Wichtig ist eine entsprechende körperliche Belastbarkeit des Patienten, um diesen multimodalen Therapieansatz möglichst gut zu tolerieren. Dabei sollte ein ASA(American Society of Anesthesiologists)-Score $\leq 3$ gefordert werden. Alle Patienten sollten, wenn möglich, vor der Operation pulmonologisch und kardiologisch abgeklärt werden. Das Alter sollte nicht über 70 Jahre sein, wobei das biologische Alter häufig nicht mit dem numerischen korreliert und das Konzept der Peritonektomie und HIPEC im Einzelfall auch beim älteren Patienten angewendet werden kann.

\section{Tumorentität und Tumorlast}

Anders als beim Mesotheliom und beim Pseudomyxoma peritonei, wo insbesondere der histologische Typ eine Rolle für die Prognose zu spielen scheint [5-8], sind beim kolorektalen Karzinom und besonders beim Magenkarzinom die intraoperative Tumorlast, beschrieben als PeritonealkarzinoseIndex (PCI), entscheidend [9]. Beim kolorektalen Karzinom besteht Konsens, dass bei einem intraoperativen PCI > 20 eine Peritonektomie in der Regel nicht indiziert ist [10-12]. Beim synchron peritoneal metastasierten Magenkarzinom gilt dies bereits bei einem PCI von 6-10 [13]. Beim metachron metastasierten Magenkarzinom ist eine zytoreduktive Chirurgie und HIPEC nicht indiziert. Allgemein gilt das Erzielen einer kompletten Zytoreduktion als prognoseführend, worunter entweder eine radikale Entfernung des gesamten Tumors (CC-0) oder das Zurücklassen von Tumorknötchen mit einer Maximalgröße von $<2,5 \mathrm{~mm}$ (CC-1) verstanden wird.

\section{Präoperative Bildgebung}

Voraussetzung für eine optimale Patientenselektion ist eine präzise präoperative Beurteilung der Lokalisation und der Ausdehnung vorhandener Tumormanifestationen [14]. Sowohl die Tumorausdehnung als auch die Lokalisation beeinflussen das postoperative Ergebnis und müssen daher bereits präoperativ möglichst genau ermittelt werden $[15,16]$. Insbesondere sollten extraabdominelle Tumormanifestationen präoperativ ausgeschlossen werden [17].

Aktuell kommen verschiedene bildgebende Verfahren in der präoperativen Diagnostik zum Einsatz. Diese beinhalten neben der Sonographie [18] die Computertomographie (CT) [19, 20], die Magnetresonanztomographie (MRT) [21] und die Positronenemissionstomographie/Computertomographie (PET/CT) [22]. Hierbei ist die kontrastangehobene CT das am weitesten verbreitete Verfahren [20]. Die Herausforderung für sämtliche bildgebende Verfahren besteht in der Unterscheidung zwischen postoperativen Veränderungen und Tumormanifestationen [23]. Dies stellt insgesamt ein Problem für
Verfahren dar, die im Wesentlichen auf der Darstellung morphologischer Veränderungen beruhen [15]. Typische Befunde bei Peritonealkarzinose beinhalten Aszites, peritoneale Raumforderungen, flächige peritoneale Verdickungen sowie Adhäsionen von viszeralem und parietalem Peritoneum [24, 25].

Verfahren, die eine weitere Gewebedifferenzierung durch zusätzliche Darstellung funktioneller Eigenschaften ermöglichen, sind die MRT und die PET/CT [22, 23]. Üblicherweise kommen bei der MRT hochauflösende T1-gewichtete kontrastangehobene 3D-Sequenzen in mehreren Phasen zum Einsatz. Ein Vorteil der dynamischen, multiphasischen, kontrastangehobenen MRT ist, dass die Akquisition mehrerer Kontrastphasen eine Beurteilung der Gewebedurchblutung und hierdurch eine Gewebecharakterisierung ermöglicht [26]. Dies und der hervorragende Weichteilkontrast der MRT tragen zur Identifizierung von Tumormanifestationen entscheidend bei [27, 28]. Studien zeigen für die MRT vielversprechende Ergebnisse mit einer guten Übereinstimmung zwischen präoperativer Bildgebung und chirurgischer Exploration hinsichtlich sowohl Tumorlokalisation als auch Ausdehnung mit diagnostischen Genauigkeiten um 90\% [29]. Eine weitere Möglichkeit der Gewebedifferenzierung mittels MRT besteht in der diffusionsgewichteten Bildgebung (diffusion-weighted imaging, DWI) [30]. Durch Veränderungen der Diffusionseigenschaften kann ebenfalls zwischen narbigen und neoplastischen Veränderungen differenziert werden.

Im Gegensatz dazu erlaubt die PET/CT mit ${ }^{18} \mathrm{~F}$-Fluordesoxyglukose (FDG) eine Aussage zur Stoffwechselaktivität von Gewebe. Das hierfür eingesetzte Radionuklid ${ }^{18} \mathrm{~F}-\mathrm{FDG}$ zeigt eine vermehrte Anreicherung in Geweben mit erhöhtem Glukosemetabolismus, was insbesondere in Tumorgewebe der Fall ist [31, 32]. Die Kombination mit der CT ermöglicht darüber hinaus eine anatomische Zuordnung und morphologische Darstellung der Tumormanifestationen. Auch für die PET/CT konnten Studien eine hohe Übereinstimmung zwischen präoperativer Bildgebung und chirurgischer Exploration zeigen [33]. Problematisch ist die Darstellung von muzinösen Tumoren in der PET/CT-Bildgebung.

Gegenüber der MRT bietet die PET/CT den weiteren Vorteil, dass hiermit der gesamte Körper erfasst wird und somit auch eine Aussage über extraabdominale Tumormanifestationen möglich ist [34].

Allen Verfahren gemeinsam ist, dass insbesondere eine diffuse rasenartige Aussaat im Rahmen einer mikronodulären Peritonealkarzinose in ihrer Ausdehnung schwer beurteilbar ist, wohingegen makronoduläre Formen besser nachgewiesen werden können [35]. So verringert sich die Sensitivität der CT bereits für Tumormanifestationen von unter $1 \mathrm{~cm}$ Durchmesser auf unter 50\% [36]. Von entscheidender Bedeutung ist die korrekte Beurteilung des Dünndarmbefalls, da hier das Ausmaß einer möglichen Resektion begrenzt ist. Um eine möglichst gute radiologische Beurteilbarkeit des Darms zu gewährleisten, ist eine gute Darmdistension durch eine negative enterale Kontrastierung, beispielsweise mittels Mannitol- 
Lösung, anzustreben. Weiterhin sollten Bewegungsartefakte durch Darmperistaltik, die einerseits zu Bildunschärfe (MRT), andererseits zu Deckungsungleichheit (PET/CT) führen, unterdrückt werden - beispielsweise durch Injektion von Butylscopolaminbromid i.v. Insbesondere Patienten mit einem mikronodulären Befallsmuster könnten im Rahmen technischer Neuerungen von einer verbesserten räumlichen Auflösung der PET-Detektoren und der MRT-Spulen sowie von der Sequenztechnologie profitieren [37]. Auch könnte eine Kombination von PET und MRT in Zukunft durch die kombinierte Darstellung von Gewebeperfusion, Diffusion und Metabolismus von Vorteil sein.

Unabhängig von der gewählten Modalität ist die Anforderung an alle bildgebenden Verfahren die korrekte präoperative Einschätzung von Lokalisation und Ausdehnung der Peritonealkarzinose und darüber hinaus das Erkennen von Tumormanifestationen, die nicht mehr vollständig resektabel sind, um ein optimales therapeutisches Vorgehen abgestimmt auf den individuellen Fall zu ermöglichen [38]. Hierfür erscheinen Verfahren, die eine Gewebecharakterisierung über eine rein morphologische Bildgebung hinaus ermöglichen, wie die dynamische, kontrastangehobene MRT und die ${ }^{18} \mathrm{~F}$ FDG PET/CT, vorteilhaft zu sein [30, 39].

\section{Diagnostische Laparoskopie}

Da gerade ein kleinnodulärer Befall des Peritoneums oder eine Infiltration der Mesenterialwurzel bei ausgedehntem Dünndarmbefall, die jeweils Kontraindikationen für eine Zytoreduktion und HIPEC darstellen, durch bildgebende Verfahren bisher nicht adäquat aufgelöst werden können, ist in jüngster Vergangenheit immer wieder die präoperative diagnostische Laparoskopie als abschließende Staging-Unter- suchung vorgeschlagen worden [40-44]. Problematisch für die diagnostische Aussagekraft der Laparoskopie sind jedoch bestehende Verwachsungen durch vorangegangene abdominalchirurgische Eingriffe, was eine exakte Dünndarmbeurteilung bzw. den Ausschluss einer Infiltration der Mesenterialwurzel ebenfalls nahezu unmöglich macht.

Ihren Stellenwert hat die Staging-Laparoskopie beim primär peritoneal metastasierten Magenkarzinom sowohl zur Bestimmung des PCI als auch im Hinblick auf die Planung einer neoadjuvant intendierten Chemotherapie zum Ausschluss einer Peritonealkarzinose. Durch die Laparoskopie wird ferner ermöglicht, Patienten mit freien intraabdominellen Tumorzellen mittels der Gewinnung einer Spülzytologie zu erkennen.

Insgesamt ist bisher die Datenlage allerdings kontrovers, sodass eine Laparoskopie bei Patienten mit Peritonealkarzinose nicht routinemäßig empfohlen werden kann.

\section{Fazit}

Trotz moderner Bildgebung ist die Abschätzung der präoperativen Tumorlast bei Patienten mit Peritonealkarzinose nach wie vor eine Herausforderung, wobei als Goldstandard die CT zu sehen ist. Eine körperliche Belastbarkeit des Patienten und eine prinzipielle Resektabilität der Peritonealkarzinose sind die Voraussetzung für die Indikationsstellung. Patienten mit einer Peritonealkarzinose sollten in einem Peritonealkarzinose-Zentrum mit entsprechender Erfahrung vorgestellt werden, um eine adäquate Patientenselektion zu gewährleisten.

\section{Disclosure Statement}

Die Autoren deklarieren keine Interessenkonflikte.

\section{Literatur}

1 Sadeghi B, Arvieux C, Glehen O, Beaujard AC, Rivoire M, Baulieux J, Fontaumard E, Brachet A Caillot JL, Faure JL, Porcheron J, Peix JL, Francois Y, Vignal J, Gilly FN: Peritoneal carcinomatosis from non-gynecologic malignancies: results of the EVOCAPE 1 multicentric prospective study. Cancer 2000;88:358-363.

2 Pyrhoenen S, Kuitunen T, Nyandoto P, Kouri M: Randomized comparison of fluorouracil, epidoxorubicin and methotrexate (FEMTX) plus supportive care with supportive care alone in patients with non-resectable gastric cancer. Br J Cancer 1995;71: 587-591.

3 Glehen O, Gilly FN, Boutitie F, Bereder JM, Quenet F, Sideris L, Mansvelt B, Lorimier G, Msika S, Elias D: Toward curative treatment of peritoneal carcinomatosis from nonovarian origin by cytoreductive surgery combined with perioperative intraperitoneal chemotherapy. A multi-institutional study of 1290 patients. Cancer 2010;116:5608-5618.
4 Yang XJ, Huang CQ, Suo T, Mei LJ, Yang GL, Cheng FL, Zhou YF, Xiong B, Yonemura Y, Li Y: Cytoreductive surgery and hyperthermic intraperitoneal chemotherapy improves survival of patients with peritoneal carcinomatosis from gastric cancer: final results of a phase III randomized clinical trial. Ann Surg Oncol 2011;18:1575-1581.

5 Yan TD, Deraco M, Baratti D, et al: Cytoreductive surgery and hyperthermic intraperitoneal chemotherapy for malignant peritoneal mesothelioma: multi-institutional experience. J Clin Oncol 2009; 27:6237-6242.

6 Elias D, Honore C, Ciuchendea R, et al: Peritoneal pseudomyxoma: results of a systematic policy of complete cytoreductive surgery and hyperthermic intraperitoneal chemotherapy. Br J Surg 2008;95: 1164-1171.

7 Bradley RF, Stewart JH 4th, Russell GB, Levine EA, Geisinger KR: Pseudomyxoma peritonei of appendiceal origin: a clinicopathologic analysis of 101 patients uniformly treated at a single institution, with literature review. Am J Surg Pathol 2006;30:551-559.
8 Sugarbaker PH: Epithelial appendiceal neoplasms. Cancer J 2009;15:225-235.

9 Jacquet P, Sugarbaker PH: Clinical research methodologies in diagnosis and staging of patients with peritoneal carcinomatosis. Cancer Treat Res 1996; 82:359-374

10 Elias D, Gilly F, Boutitie F, et al: Peritoneal colorectal carcinomatosis treated with surgery and perioperative intraperitoneal chemotherapy: retrospective analysis of 523 patients from a multicentric French study. J Clin Oncol 2010;28:63-68.

11 da Silva RG, Sugarbaker PH: Analysis of prognostic factors in seventy patients having a complete cytoreduction plus perioperative intraperitoneal chemotherapy for carcinomatosis from colorectal cancer. J Am Coll Surg 2006;203:878-886.

12 Glehen O, Kwiatkowski F, Sugarbaker PH, et al Cytoreductive surgery combined with perioperative intraperitoneal chemotherapy for the management of peritoneal carcinomatosis from colorectal cancer: a multi-institutional study. J Clin Oncol 2004;22:3284-3292. 
13 Yonemura Y, Elnemr A, Endou Y, et al: Multidisciplinary therapy for treatment of patients with peritoneal carcinomatosis from gastric cancer. World J Gastrointest Oncol 2010;2:85-97.

14 Koenigsrainer I, Aschoff P, Zieker D, Beckert S, Glatzle J, Pfannenberg C, Miller S, Hartmann JT, Schroeder TH, Bruecher BL, Koenigsrainer A Selection criteria for peritonectomy with hyperthermic intraoperative chemotherapy (HIPEC) in peritoneal carcinomatosis. Zentralbl Chir 2008;133: 468-472.

15 Yan TD, Sim J, Morris DL: Selection of patients with colorectal peritoneal carcinomatosis for cytoreductive surgery and perioperative intraperitoneal chemotherapy. Ann Surg Oncol 2007;14:18071817.

16 Quayyum A, Coakley FV, Westphalen AC, et al: Role of CT and MRI in predicting optimal cytoreduction of newly diagnosed primary epithelial ovarian cancer. Gyneco Oncol 2005;96:301-306.

17 Glehen O, Mohamed F, Gilly FN: Peritoneal carcinomatosis from digestive tract cancer: new management by cytoreductive surgery and intraperitoneal chemohyperthermia. Lancet Oncol 2004;5: 219-228.

-18 Testa A, Ludovisi M, Mascilini F, Di Legge A, Malaggese M, Faqotti A, Fanfani F, Salerno M, Ercoli A, Scambia G, Ferrandina G: Ultrasound evaluation of intra-abdominal sites of disease to predict the likelihood of suboptimal cytoreduction in advanced ovarian cancer: a prospective study. Ultrasound Obstet Gynecol 2012;39:99-105.

19 Funicelli L, Travaini LL, Landoni F, Trifiro G, Bonello L, Bellomi M: Peritoneal carcinomatosis from ovarian cancer: the role of CT and $\left[{ }^{18} \mathrm{~F}\right] \mathrm{FDG}-$ PET/CT. Abdom Imaging 2010;35:701-707.

20 Forstner R: Radiological staging of ovarian cancer: imaging findings and contribution of $\mathrm{CT}$ and MRI Eur Radiol 2007;17:3223-3246.

21 Low RN, Barone RM, Lacey C, Sigeti JS, Alzate GD, Sebrechts CP: Peritoneal tumor: MR imaging with dilute oral barium and intravenous gadolinium containing contrast agents compared with unenhanced MR imaging and CT. Radiology 1997; 204:513-520.

-22 Pfannenberg C, Koenigsrainer I, Aschoff P, Oeksuez MO, Zieker D, Beckert S, Symons S, Nieselt K, Glatzle J, Weyhern CV, Brücher BL, Claussen CD, Koenigsrainer A: (18)F-FDG-PET/CT to select patients with peritoneal carcinomatosis for cytoreductive surgery and hyperthermic intraperitoneal chemotherapy. Ann Surg Oncol 2009;16:1295-1303.
23 Iafrate F, Ciolina M, Sammartino P, Baldassari P, Rengo M, Lucchesi P, Sibio S, Accarpio F, Di Giorgio A, Laghi A: Peritoneal carcinomatosis: imaging with 64-MDCT and 3T MRI with diffusion-weighted imaging. Abdom Imaging 2012;37:616-627.

24 Souza FF, Jagganathan J, Ramayia N, Cirian J, Jackman D, Van den Abbeele A, Ros PR: Recurrent malignant peritoneal mesothelioma: radiological manifestations. Abdom Imaging 2010;35:315321.

25 Morita H, Aoki J, Taketomi A, Sato N, Endo K: Serous surface papillary carcinoma of the peritoneum: clinical, radiologic and pathologic findings in 11 patients. AJR Am J Roentgenol 2004;183:923-928.

26 Low RN, Sigeti JS: MR imaging of peritoneal disease: comparison of contrast enhanced fast multiplanar spoiled gradient recalled and spin echo imaging. AJR 1994;163:1131-1140.

27 Forstner R, Hricak H, Powell CB, et al: Ovarian cancer recurrence: value of MR imaging. Radiology 1995;196:715-720.

28 Low RN, Carter WD, Saleh F, et al: Ovarian cancer: comparison of findings with perfluorocarbonenhanced MR imaging, In-111-CYT-103 immunoscintigraphy, and CT. Radiology 1995;195:391-400.

29 Klumpp BD, Aschoff P, Schwenzer N, Fenchel M, Koenigsrainer I, Falch C, Bruecher B, Claussen CD, Koenigsrainer A, Pfannenberg C, Kramer U, Miller S: Peritoneal carcinomatosis: comparison of dynamic contrast-enhanced magnetic resonance imaging with surgical and histopathologic findings. Abdom Imaging 2012;37:834-842.

30 Sato Y, Ichikawa T, Motosugi U, Kimura K, Sou H, Sano K, Araki T: Diagnosis of peritoneal dissemination: comparison of ${ }^{18} \mathrm{~F}-\mathrm{FDG} \mathrm{PET} / \mathrm{CT}$, diffusion-weighted MRI and contrast-enhanced MDCT. AJR Am J Roentgenol 2011;196:447-453.

31 Glaspy JA, Hawkins R, Hoh CK, Phelps ME: Use of positron emission tomography in oncology. Oncology 1993;7:41-55.

32 Kole AC, Nieweq OE, Pruim J, Hoekstra HJ, Koops HS, Roodenburg JL, Vaalburg W, Vermey A: Detection of unknown occult primary tumors using positron emission tomography. Cancer 1998; 82:1160-1166.

33 Klumpp BD, Schwenzer N, Aschoff P, Miller S, Kramer U, Claussen CD, Bruecher B, Koenigsrainer A, Pfannenberg C: Preoperative assessment of peritoenal carcinomatosis: intraindividual comparison of 18F-FDG PET/CT and MRI. Abdom Imaging 2013;38:64-71.
4 Rakheja R, Makis W, Hickeson M: Extraovarian primary peritoneal carcinoma: staging with $18 \mathrm{~F}$ FDG PET/CT. Abdom Imaging 2012;37:304-308.

35 Low RN: MR imaging of the peritoneal spread of malignancy. Abdom Imaging 2007;32:267-283.

66 Zissin R, Hertz M, Shapiro-Feinberg M, et al: Primary serous papillary carcinoma of the peritoneum: CT findings. Clin Radiol 2001;56:740-745.

37 De Iaco P, Musto A, Orazi L, Zamagni C, Rosati M, Allegri V, Cacciari N, Al-Nahhas A, Rubello D, Venturoli S, Fanti S: FDG-PET/CT in advanced ovarian cancer staging: value and pitfalls in detecting lesions in different abdominal and pelvic quadrants compared with laparoscopy. Eur J Radiol 2011;80:e98-103.

38 Dromain C, Leboulleux S, Auperin A, Goere D, Malka D, Lumbroso J, Schumberger M, Sigal R, Elias D: Staging of peritoneal carcinomatosis: enhanced CT vs. PET/CT. Abdom Imaging 2008;33: 87-93.

39 Priest AN, Gill AB, Kataoka M, McLean MA, Joubert I, Graves MJ, Griffiths JR, Crawford RAF, Earl H, Brenton JD, Lomas DJ, Sala E: Dynamic contrast-enhanced MRI in ovarian cancer: initial experience at 3 tesla in primary and metastatic disease. Magn Reson Med 2010;63:1044-1049.

40 Garofalo A, Valle M: Laparoscopy in the management of peritoneal carcinomatosis. Cancer J 2009; 15:190-195.

41 Pomel C, Appleyard TL, Gouy S, Rouzier R, Elias D: The role of laparoscopy to evaluate candidates for complete cytoreduction of peritoneal carcinomatosis and hyperthermic intraperitoneal chemotherapy. Eur J Surg Oncol 2005;31:540-543.

42 Laterza B, Kusamura S, Baratti D, Oliva GD, Deraco M: Role of explorative laparoscopy to evaluate optimal candidates for cytoreductive surgery and hyperthermic intraperitoneal chemotherapy (HIPEC) in patients with peritoneal mesothelioma. In Vivo 2009;23:187-190.

43 Valle M, Federici O, Garofalo A: Patient selection for cytoreductive surgery and hyperthermic intraperitoneal chemotherapy, and role of laparoscopy in diagnosis, staging, and treatment. Surg Oncol Clin N Am 2012;21:515-531.

44 Iversen LH, Rasmussen PC, Laurberg S: Value of laparoscopy before cytoreductive surgery and hyperthermic intraperitoneal chemotherapy for peritoneal carcinomatosis. Br J Surg 2013;100:285-292. 